\title{
Active learning surrogate models for the conception of systems with multiple failure modes.
}

\author{
- ADMOS 2015 - \\ G. Perrin* \\ * Commissariat aux Energies Nucléaires (CEA) \\ CEA/DAM/DIF, F-91297, Arpajon, France \\ e-mail: guillaume.perrin2@cea.fr
}

\begin{abstract}
The conception (or risk assessment) of complex mechanical systems has to take into account a series of constraints. Such constraints can be due to certification criteria, performance objectives, cost limitations, and so on. In this context, the role of simulation has kept increasing for the last decades, as it should be able to predict if a given configuration of the system is likely to fulfill these constraints without having to build it and to test it experimentally. In many cases, the computation of these constraints is associated with a series of computer software, whose physics can vary a lot. For instance, in the car industry, the conception of a new vehicle can be subjected to constraints on its size and weight, which are rather easy to compute, but also on its emergency stopping distance, its crash or aerodynamic resistance, which can be much more difficult to evaluate. Thus, several software (structure dynamics, multibody modeling, fluid dynamics software for instance) are generally needed to verify all the constraints.

Numerical methods to identify the limits of such "conception domains", that is to say domains in which all the constraints are verified, are needed [1]. As the computational costs associated with the constraints can be very different, the question then arises of how to optimize the number of evaluations of each code to minimize the uncertainties about these limits, for a given computational budget.

In this prospect, this paper presents an innovative approach to predict the limits of the considered conception domain, but also to quantify the uncertainty associated with this prediction. Based on an adaptive Gaussian process regression, this method allows us to find iteratively the new code evaluations that will maximize the knowledge about the searched limits at the minimal computational cost.
\end{abstract}

First, the scientific basis of such an approach will be presented. The efficiency of such a method will then be illustrated on an analytical example.

\section{REFERENCES}

[1] W. Fauriat, N. Gayton, "AK-SYS: An adaptation of the AK-MCS method for system reliability”, Reliability Engineering and System Safety, Vol. 123, pp. 137-144, (2014). 